PROCEEDINGS OF THE

AMERICAN MATHEMATICAL SOCIETY

Volume 132, Number 2, Pages 579-586

S 0002-9939(03)07068-0

Article electronically published on September 5, 2003

\title{
MINIMAL 2-COMPLEXES AND THE D(2)-PROBLEM
}

\author{
F. E. A. JOHNSON
}

(Communicated by Ronald A. Fintushel)

\begin{abstract}
We show that when $n \geq 5$ there is a minimal algebraic 2-complex over the quaternion group $Q\left(2^{n}\right)$ which is not homotopy equivalent to the Cayley complex of the standard minimal presentation. This raises the possibility that Wall's D(2)-property might fail for $Q\left(2^{n}\right)$.
\end{abstract}

\section{INTRODUCTION}

Let $G$ be a group with a finite presentation $\mathcal{G}=\left\langle x_{1}, \ldots, x_{g} \mid W_{1}, \ldots, W_{r}\right\rangle$, and let $X=X_{\mathcal{G}}$ be the associated Cayley complex of $\mathcal{G}$. We interpret the cellular chain complex of the universal cover $\tilde{X}$,

$$
C_{*}(\mathcal{G})=\left(0 \rightarrow \pi_{2}(X) \rightarrow C_{2}(\tilde{X}) \stackrel{\partial_{2}}{\rightarrow} C_{1}(\tilde{X}) \stackrel{\partial_{1}}{\rightarrow} C_{0}(\tilde{X}) \stackrel{\epsilon}{\rightarrow} \mathbf{Z} \rightarrow 0\right),
$$

as an exact sequence of right $\mathbf{Z}[G]$-modules, the second homotopy group $\pi_{2}(X)$ being identified by the Hurewicz Theorem with $\operatorname{Ker}\left(\partial_{2}: C_{2}(\tilde{X}) \rightarrow C_{1}(\tilde{X})\right)$. More generally, by an algebraic 2-complex over $G$ we mean an exact sequence of $\mathbf{Z}[G]$ modules of the form

$$
\mathbf{E}=\left(0 \rightarrow J \rightarrow E_{2} \rightarrow E_{1} \rightarrow \quad E_{0} \rightarrow \mathbf{Z} \rightarrow 0\right)
$$

where $E_{r}$ is finitely generated free over $\mathbf{Z}[G]$ for $0 \leq r \leq 2$. The question which concerns us may be posed conveniently in the following form:

Realization Problem. Let $G$ be a finitely presented group. Is every algebraic 2-complex over $G$ realized up to chain homotopy in the form $C_{*}(\mathcal{G})$ for some finite presentation $\mathcal{G}$ of $G$ ?

In the algebraic 2-complex $\mathbf{E}=\left(0 \rightarrow J \rightarrow E_{2} \rightarrow E_{1} \rightarrow E_{0} \rightarrow \mathbf{Z} \rightarrow 0\right)$ the module $J$ plays the role of an 'algebraic $\pi_{2}$ '. In this paper we consider only the case where $G$ is finite; then there is a well-defined stable module $\Omega_{3}(\mathbf{Z})$ which contains all such possible algebraic homotopy groups $J$. E is minimal when $\operatorname{rk}_{\mathbf{Z}}(J)$ attains the minimum possible value within $\Omega_{3}(\mathbf{Z})$. Browning's Theorem ([1]) essentially shows that the Realization Problem is equivalent to the problem of realizing minimal 2complexes ([1], [4]; see also [6]). Let $Q(4 m)$ denote the quaternion group of order $4 m$, given in the following standard presentation:

$$
\mathcal{Q}(4 m)=\left\langle x, y \mid x^{m}=y^{2} ; x y x=y\right\rangle .
$$

Received by the editors December 28, 2000 and, in revised form, August 22, 2002.

2000 Mathematics Subject Classification. Primary 55M05, 57M20; Secondary 16D70.

Key words and phrases. Algebraic 2-complexes, non-cancellation, minimal presentations.

(C)2003 American Mathematical Society 
The Cayley complex of the presentation $\mathcal{Q}(4 m)$ determines a minimal 2-complex. We show:

Theorem A. For each $n \geq 5$ there exists a minimal algebraic 2-complex $\mathcal{X}_{*}(n)$ over $Q\left(2^{n}\right)$ such that $\mathcal{X}_{*}(n)$ is not chain homotopy equivalent to $C_{*}\left(\mathcal{Q}\left(2^{n}\right)\right)$, the Cayley complex of the standard presentation.

In his study of the homotopy properties of finite complexes [10], Wall encountered the following fundamental problem which relates dimensions 2 and 3 :

$\mathbf{D}(2)$-problem. Let $X^{(3)}$ be a finite 3-complex which is cohomologically twodimensional in the sense that $H^{3}\left(X^{(3)} ; \mathcal{B}\right)=0$ for all local coefficient systems $\mathcal{B}$; is it true that $X^{(3)}$ is homotopy equivalent to a finite 2 -dimensional complex?

In [6], we showed that, for finite fundamental groups $G$, the $\mathrm{D}(2)$-problem is equivalent to the Realization Problem. The question of whether $\mathcal{X}_{*}(n)$ is realizable up to homotopy as the Cayley complex of some finite presentation is an intriguing one. Any such presentation would necessarily be balanced, but homotopically distinct from the standard presentation. Indeed, its Cayley complex could not be equivalent to the wedge of the standard Cayley complex with that of any 'exotic' presentation of the trivial group 1 However, if no such presentation exists, then the status of the complexes $\mathcal{X}_{*}(n)$ is perhaps even more interesting.

Theorem B. For each $n \geq 5$, either $Q\left(2^{n}\right)$ possesses a balanced presentation which is homotopically distinct from the standard presentation or else the $\mathrm{D}(2)$-property fails for $Q\left(2^{n}\right)$.

\section{Stable modules ANd tree structures}

$G$ will denote a finite group, and $\mathcal{F}(\mathbf{Z}[G])$ the category of right $\mathbf{Z}[G]$-lattices; that is, right $\mathbf{Z}[G]$-modules which are finitely generated and free as modules over Z. We denote by ' $\sim$ ' the stability relation on $\mathcal{F}(\mathbf{Z}[G])$ :

$$
M_{1} \sim M_{2} \Longleftrightarrow M_{1} \oplus \mathbf{Z}[G]^{n_{1}} \cong M_{2} \oplus \mathbf{Z}[G]^{n_{2}} \text { for some } n_{1}, n_{2} \geq 0 .
$$

If $M \in \mathcal{F}(\mathbf{Z}[G])$-lattice, its associated stable module $[M]$ is the class of $\mathbf{Z}[G]$-lattices equivalent to $M$ under $\sim$. 'Schanuel's Lemma' [7] shows that, for each module $M \in \mathcal{F}(\mathbf{Z}[G])$, there is a well-defined stable module $\Omega_{n}(M)$ consisting of modules stably equivalent to a module $J$ which occurs in an exact sequence of the form

$$
0 \rightarrow J \rightarrow F_{n-1} \rightarrow \ldots \rightarrow F_{0} \rightarrow M \rightarrow 0
$$

where each $F_{i}$ is finitely generated free. Clearly $\Omega_{1}(\mathbf{Z})$ is the stable class of the augmentation ideal $\mathbf{I}(G)$ of $G$.

Any stable module $[M]$ has a representation as an infinite tree in which the vertices are the modules $N \in[M]$, with an arrow $N \rightarrow N \oplus \mathbf{Z}[G]$ for each isomorphism type $N$. By the Swan-Jacobinski Theorem (see (2.1) of [5]), $\Omega_{2 n+1}(\mathbf{Z})$ has the form of a fork; that is, a locally finite tree with only one vertex at each positive integral height, and a finite set of vertices at height zero (Figure 1). The height function is obtained by an appropriate normalisation of Z-rank. Modules at level zero, the so-called root modules $M_{0}$, have the property that any module $N \in[M]$ takes the form $N \cong M_{0} \oplus \mathbf{Z}[G]^{n}$. A stable module is said to be straight when it is isomorphic, as a tree, to the natural numbers $\mathbf{N}$; that is, when there is only one isomorphism type at level 0 . The notion behaves well under duality. Let $M \in \mathcal{F}(\mathbf{Z}[G])$; then:

\footnotetext{
${ }^{1}$ This formulation was suggested to the author by Raymond Lickorish.
} 


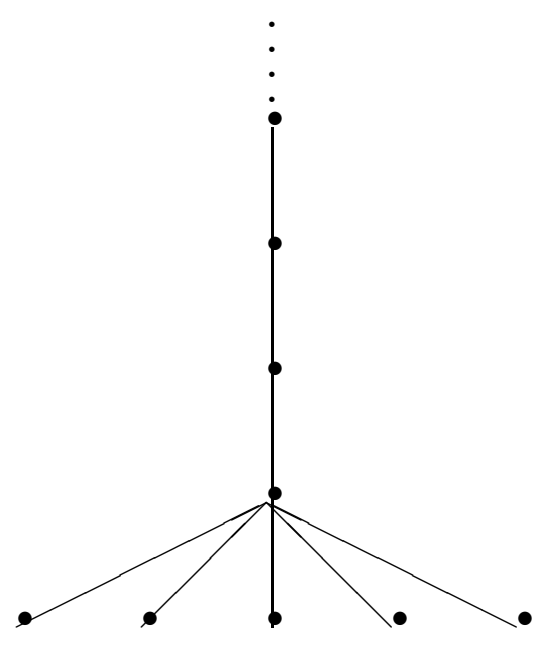

FiguRE 1.

Proposition 1.1. $[M]$ is straight $\Longleftrightarrow\left[M^{*}\right]$ is straight.

$G$ is said to be $n$-straight when the stable module $\Omega_{n}(\mathbf{Z})$ over $\mathbf{Z}[G]$ is straight. The finite group $G$ has the cancellation property for free modules when finitely generated stably free modules over $\mathbf{Z}[G]$ are free; that is, when an isomorphism of the form $B \oplus \mathbf{Z}[G]^{\beta} \cong \mathbf{Z}[G]^{\alpha}$ implies that $B \cong \mathbf{Z}[G]^{\alpha-\beta}$. In [5] (Prop. 2.7) we showed:

Proposition 1.2. If the finite group $G$ has the cancellation property for free modules, then $G$ is 1-straight.

We will show that under certain conditions the converse is also true.

\section{Root MOdULES AND THE WEAK CANCELLATION PROPERTY}

We are concerned with stably free $\mathbf{Z}[G]$ modules of $\mathbf{Z}[G]$-rank $=1$; that is, $\mathbf{Z}[G]$-lattices $\mathbf{S}$ with the property that $\mathbf{S} \oplus \mathbf{Z}[G] \cong \mathbf{Z}[G] \oplus \mathbf{Z}[G]$. The consequences of this are explored in $\S 4$. By Wedderburn's Theorem, for such a module $\mathbf{S}$ there is an isomorphism of $\mathbf{Q}[G]$-modules $\varphi: \mathbf{S} \otimes \mathbf{Q} \stackrel{\simeq}{\rightarrow} \mathbf{Q}[G]$. Let $\epsilon: \mathbf{Q}[G] \rightarrow \mathbf{Q}$ be the augmentation map, and let $\xi: \mathbf{S} \rightarrow \mathbf{Q}$ be the mapping

$$
\xi(\mathbf{x})=\epsilon \circ \varphi(\mathbf{x} \otimes 1) \text {. }
$$

Put $\mathbf{A}=\operatorname{Im}(\xi) \subset \mathbf{Q}$. Since $\mathbf{A}$ is a nontrivial finitely generated subgroup of $\mathbf{Q}$, there is a group isomorphism $\psi: \mathbf{A} \stackrel{\simeq}{\rightrightarrows} \mathbf{Z}$. On taking $\eta=\psi \circ \xi$ we obtain:

Proposition 2.1. Let $\mathbf{S}$ be a stably free $\mathbf{Z}[G]$-module of $\mathbf{Z}[G]$-rank $=1$; then there exists a surjective $\mathbf{Z}[G]$-homomorphism $\eta: \mathbf{S} \rightarrow \mathbf{Z}$.

We observed in $\S 1$ that $\Omega_{1}(\mathbf{Z})=[\mathbf{I}(G)]$; it follows that $J \in \mathcal{F}(\mathbf{Z}[G])$ is a root module of $\Omega_{1}(\mathbf{Z})$ precisely when $J \oplus \mathbf{Z}[G] \cong \mathbf{I}(G) \oplus \mathbf{Z}[G]$. Less obviously, we have:

Proposition 2.2. A $\mathbf{Z}[G]$-lattice $J$ is a root module of $\Omega_{1}(\mathbf{Z})$ precisely when there exists an exact sequence $0 \rightarrow J \rightarrow \mathbf{S} \rightarrow \mathbf{Z} \rightarrow 0$ where $\mathbf{S}$ is a stably free $\mathbf{Z}[G]$ module of $\mathbf{Z}[G]$-rank $=1$. 
Proof. An easy argument using double dualisation and the universal property of free modules shows that if $F$ is a finitely generated free module over $\mathbf{Z}[G]$ ), then $F$ is injective relative to $\mathcal{F}(\mathbf{Z}[G])$. Since $J$ is assumed to be a root module for $\Omega_{1}(\mathbf{Z})$, then $J \oplus \mathbf{Z}[G] \cong \mathbf{I}(G) \oplus \mathbf{Z}[G]$. However $\mathbf{I}(G) \oplus \mathbf{Z}[G]$ occurs in an exact sequence

$$
0 \rightarrow \mathbf{I}(G) \oplus \mathbf{Z}[G] \rightarrow \mathbf{Z}[G] \oplus \mathbf{Z}[G] \rightarrow \mathbf{Z} \rightarrow 0,
$$

so that, by factoring out a copy of $\mathbf{F} \cong \mathbf{Z}[G]$, we obtain an extension

$$
0 \rightarrow J \rightarrow(\mathbf{Z}[G] \oplus \mathbf{Z}[G]) / \mathbf{F} \rightarrow \mathbf{Z} \rightarrow 0 .
$$

Put $\mathbf{S}=(\mathbf{Z}[G] \oplus \mathbf{Z}[G]) / \mathbf{F}$; clearly $\mathbf{S}$ is torsion free and so is a $\mathbf{Z}[G]$-lattice. As we have noted, since $\mathbf{F}$ is free, it is relatively injective, and the exact sequence $0 \rightarrow \mathbf{F} \rightarrow \mathbf{Z}[G] \oplus \mathbf{Z}[G] \rightarrow \mathbf{S} \rightarrow 0$ of $\mathbf{Z}[G]$-lattices splits. Thus $\mathbf{Z}[G] \oplus \mathbf{Z}[G] \cong$ $\mathbf{S} \oplus \mathbf{F} \cong \mathbf{S} \oplus \mathbf{Z}[G]$.

If $G$ is a finite group and $n$ is a positive integer, we denote by $(\mathbf{I}, n)$ the (twosided) ideal of $\mathbf{Z}[G]$ consisting of elements of augmentation divisible by $n$; that is, $(\mathbf{I}, n)=\epsilon^{-1}(\mathbf{n})$ where $\epsilon: \mathbf{Z}[G] \rightarrow \mathbf{Z}$ is the augmentation map, and $(\mathbf{n}) \subset \mathbf{Z}$ is the ideal generated by $n$. The modules $(\mathbf{I}, n)$ are called Swan modules. $(\mathbf{I}, n)$ occurs in an extension $\mathcal{E}(n)=(0 \rightarrow \mathbf{I}(G) \rightarrow(\mathbf{I}, n) \rightarrow \mathbf{Z} \rightarrow 0)$ which, by Yoneda Theory, corresponds to an extension class or ' $k$-invariant' $k(\mathcal{E}(n)) \in \operatorname{Ext}^{1}(\mathbf{Z}, \mathbf{I}(G)) \cong \mathbf{Z} /|G|$, in such a way that $k(\mathcal{E}(n))$ corresponds to $[n] \in \mathbf{Z} /|G|$. The dual module $\mathbf{I}^{*}(G)$ may be identified with the quotient ring $\mathbf{Z}[G] /(\Sigma)$ where $\Sigma=\sum_{g \in G} g$, so there is a natural surjective ring homomorphism $\eta: \mathbf{I}^{*}(G) \rightarrow \mathbf{Z} /|G|$. The following are known $([8])$ :

(A) $\mathcal{E}(m)$ is congruent to $\mathcal{E}(n)$ if and only if $m \equiv n(\bmod |G|)$;

(B) $(\mathbf{I}, n)$ is projective if and only if $[n] \in(\mathbf{Z} /|G|)^{*}$;

(C) $(\mathbf{I}, m) \cong(\mathbf{I}, n)$ if and only if there exists a unit $u \in \mathbf{I}^{*}(G)$ such that $\eta(u) m=$ $n$.

We say that $G$ has the weak cancellation property when for each $[n] \in(\mathbf{Z} /|G|)^{*}$ either $(\mathbf{I}, n)$ is free or $(\mathbf{I}, n)$ is not stably free.

Proposition 2.3. Let $G$ be a finite group with the weak cancellation property, and let $\mathcal{E}=(0 \rightarrow J \rightarrow \mathbf{S} \rightarrow \mathbf{Z} \rightarrow 0)$ be an extension in which $\mathbf{S}$ is a stably free $\mathbf{Z}[G]$-module of $\mathbf{Z}[G]$-rank $=1$. If $\mathbf{S}$ is not free, then $J \not \mathbf{I}(G)$.

Proof. Let $\mathcal{E}=(0 \rightarrow J \rightarrow \mathbf{S} \rightarrow \mathbf{Z} \rightarrow 0)$ be an extension with $\mathbf{S}$ projective. Suppose that $\varphi: J \rightarrow \mathbf{I}(G)$ is an isomorphism. Write $\varphi_{*}(\mathcal{E})=(0 \rightarrow \mathbf{I}(G) \rightarrow \mathbf{T} \rightarrow \mathbf{Z} \rightarrow 0)$. By functoriality, $\mathbf{T} \cong \mathbf{S}$. Put $r=k\left(\varphi_{*}(\mathcal{E})\right)$. Then $\mathbf{S} \cong \mathbf{T} \cong(\mathbf{I}, r)$, and since $\mathbf{S}$ is projective, $r$ is coprime to $|G|$. Now suppose that $\mathbf{S}$ is stably free. Then $(\mathbf{I}, r)$ is also stably free. But $G$ has the weak cancellation property, so that $(\mathbf{I}, r)$ is free, and hence $\mathbf{S}$ is also free. Thus we have shown that if $J \cong \mathbf{I}(G)$ and $\mathbf{S}$ is stably free, then $\mathbf{S} \cong \mathbf{Z}[G]$. In the contrapositive, if $\mathbf{S}$ is stably free and $\mathbf{S} \neq \mathbf{Z}[G]$, then $J \neq \mathbf{I}(G)$.

Corollary 2.4. Let $G$ be a finite group with the weak cancellation property. Then $G$ is 1-straight $\Leftrightarrow G$ has the cancellation property for free modules.

A root module $J$ of $\Omega_{1}(\mathbf{Z})$ is said to be exotic when $J \neq \mathbf{I}(G)$. Swan [8] shows that for $n \geq 5$, the quaternion groups $Q\left(2^{n}\right)$ have the weak cancellation property but not the cancellation property for free modules; thus they possess exotic root modules. 


\section{Algebraic 2-complexes}

Let $\mathbf{E}=\left(0 \rightarrow J \rightarrow E_{2} \rightarrow E_{1} \rightarrow E_{0} \rightarrow \mathbf{Z} \rightarrow 0\right)$ be an algebraic 2-complex over $\mathbf{Z}[G]$; by analogy with the geometric case, we think of $J$ as being an 'algebraic $2^{\text {nd }}$ homotopy group' and write $J=\pi_{2}(\mathbf{E})$. Clearly $J \in \Omega_{3}(\mathbf{Z})$. We denote by $\operatorname{Alg}_{G}(J)$ the class of algebraic 2-complexes over $\mathbf{Z}[G]$ for which $\pi_{2}=J$; moreover we denote by $\mathbf{A l g}_{G}$ the set of homotopy types of algebraic 2-complexes over $\mathbf{Z}[G]$, and by $\mathcal{H}_{G}$ the category whose objects are finite 2-dimensional cell complexes $K$ with $\pi_{1}(K)=G$ and whose morphisms are cellular homotopy classes of cellular maps. If $K \in \mathcal{H}_{G}$, the cellular chain complex

$$
\mathcal{C}(K)=\left(0 \rightarrow \pi_{2}(K) \rightarrow C_{2}(\tilde{K}) \rightarrow C_{1}(\tilde{K}) \rightarrow C_{0}(\tilde{K}) \rightarrow \mathbf{Z} \rightarrow 0\right)
$$

defines an object in $\operatorname{Alg}_{G}\left(\pi_{2}(K)\right)$ and gives a mapping $\mathcal{C}: \mathcal{H}_{G} \rightarrow \mathbf{A l g}_{G}$ which preserves homotopy type. There is a stabilization process within $\mathcal{H}_{G}$, namely $X \mapsto$ $X \vee S^{2}$, and we impose the structure of a directed graph on the objects of $\mathcal{H}_{G}$ by drawing an arrow $X \rightarrow X \vee S^{2}$. It is straightforward to see that $\mathcal{H}_{G}$ is a tree; this observation is originally due to Dyer and Sieradski [2]. Likewise, a tree structure is introduced into $\mathbf{A l g}_{G}$ by means of the algebraic analogue of this stabilization process, namely

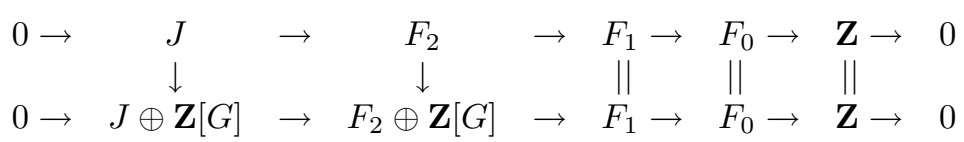

In his thesis [1] (see also [4]), Browning proved the following Stability Theorem, which generalizes the Swan-Jacobinski Theorem to this setting:

Theorem 3.1 (Browning). Let $G$ be a finite group, and let $J \in \Omega_{3}(\mathbf{Z})$. Then for any $\mathcal{E}, \mathcal{E}^{\prime} \in \operatorname{Alg}_{G}(J \oplus \mathbf{Z}[G])$, there exists a weak homotopy equivalence $h: \mathcal{E} \rightarrow \mathcal{E}^{\prime}$.

In particular, $\operatorname{Alg}_{G}(J)$ consists of a single element provided $J \in \Omega_{3}(\mathbf{Z})$ is not algebraically minimal, and so $\mathbf{A l g}_{G}$ is actually a fork. For each module $J \in \Omega_{3}(\mathbf{Z})$, the congruence classes of elements in $\operatorname{Ext}^{3}(\mathbf{Z}, J)$ are parametrized by the group $\operatorname{Ext}_{\mathbf{Z}[G]}^{3}\left(\mathbf{Z}, \Omega_{3}(\mathbf{Z})\right) \cong \mathbf{Z} /|G|$. A fortiori, when $J$ is algebraically minimal, the number of weak homotopy types of elements in $\operatorname{Alg}_{G}(J)$ is finite. Furthermore, since the number of isomorphism types of algebraically minimal modules is also finite, we see that:

Corollary 3.2. Let $G$ be a finite group. Then $\mathbf{A l g}_{G}$, the tree of chain homotopy types of algebraic 2-complexes, is a fork with a finite number of prongs.

The functor 'algebraic $\pi_{2}$ ' defines a morphism of directed graphs

$$
\pi_{2}: \mathbf{A l g}_{G} \rightarrow \Omega_{3}(\mathbf{Z}) .
$$

Proposition 3.3. For any finite group $G$, the mapping $\pi_{2}: \mathbf{A l g}_{G} \rightarrow \Omega_{3}(\mathbf{Z})$ is surjective.

Proof. Suppose that $J \in \Omega_{3}(M)$; then there exists a module $D$ such that for some $n, m \geq 0, J \oplus \mathbf{Z}[G]^{m} \cong D \oplus \mathbf{Z}[G]^{n}$, and such that $D$ occurs in an exact sequence

$$
0 \rightarrow D \rightarrow \mathbf{Z}[G]^{\gamma} \rightarrow \mathbf{Z}[G]^{\beta} \rightarrow \mathbf{Z}[G]^{\alpha} \rightarrow \mathbf{Z} \rightarrow 0 .
$$

Thus $J \oplus \mathbf{Z}[G]^{m}$ occurs in an exact sequence

$$
0 \rightarrow J \oplus \mathbf{Z}[G]^{m} \rightarrow \mathbf{Z}[G]^{\gamma+n} \rightarrow \mathbf{Z}[G]^{\beta} \rightarrow \mathbf{Z}[G]^{\alpha} \rightarrow \mathbf{Z} \rightarrow 0 .
$$


We split this last sequence as follows:

$$
\begin{gathered}
0 \rightarrow J \oplus \mathbf{Z}[G]^{m} \rightarrow \mathbf{Z}[G]^{\gamma+n} \rightarrow K \rightarrow 0 ; \\
0 \rightarrow K \rightarrow \mathbf{Z}[G]^{\beta} \rightarrow \mathbf{Z}[G]^{\alpha} \rightarrow \mathbf{Z} \rightarrow 0 .
\end{gathered}
$$

Dividing through (I) by $\mathbf{Z}[G]^{m}$ gives an extension

$$
0 \rightarrow J \rightarrow \mathbf{Z}[G]^{\gamma+n} / \mathbf{Z}[G]^{m} \rightarrow K \rightarrow 0 .
$$

From the form of (II), since $K$ is torsion free over $\mathbf{Z}$, then $\mathbf{Z}[G]^{\gamma+n} / \mathbf{Z}[G]^{m}$ is also. As in the proof of $(2.2), \mathbf{Z}[G]^{m}$ is injective relative to $\mathcal{F}(\mathbf{Z}[G])$, so that $S=\mathbf{Z}[G]^{\gamma+n} / \mathbf{Z}[G]^{m}$ is stably free over $\mathbf{Z}[G]$. Splicing (II) with (III) gives an extension

$$
0 \rightarrow J \rightarrow S \rightarrow \mathbf{Z}[G]^{\beta} \rightarrow \mathbf{Z}[G]^{\alpha} \rightarrow \mathbf{Z} \rightarrow 0 .
$$

If $S$ is free over $\mathbf{Z}[G]$ we may take $\mathbf{E}$ to be this last sequence. If $S$ is not free over $\mathbf{Z}[G]$, then $S \oplus \mathbf{Z}[G]^{c} \cong \mathbf{Z}[G]^{d}$ so that we may take $\mathbf{E}$ to be the obvious exact sequence of the form

$$
0 \rightarrow J \rightarrow \mathbf{Z}[G]^{d} \rightarrow \mathbf{Z}[G]^{\beta+c} \rightarrow \mathbf{Z}[G]^{\alpha} \rightarrow \mathbf{Z} \rightarrow 0
$$

and this completes the proof.

\section{A Candidate For the failure of the D(2)-Property}

The modules $(\mathbf{I}, r)$ over a variety of finite groups have been extensively studied by Swan. In particular, he shows (8] Theorem (17.7)) that over the quaternion group $Q\left(2^{n}\right)$ for $n \geq 3$ there only two isomorphism classes amongst the modules $(\mathbf{I}, r)$; when $r= \pm 1(\bmod 8),(\mathbf{I}, r)$ is free; by contrast, for $r= \pm 3(\bmod 8)$ the modules $(\mathbf{I}, r)$ constitute a single isomorphism class, which is nonfree, and which generates the projective class group $\tilde{K}_{0}\left(\mathbf{Z}\left[Q\left(2^{n}\right)\right]\right) \cong \mathbf{Z} / 2 \mathbf{Z}$. It follows easily that:

Theorem 4.1 (Swan). For any $n \geq 3$, the quaternion group $Q\left(2^{n}\right)$ of order $2^{n}$ has the weak cancellation property.

Extending Vigneras' work [9], Swan has also shown ([8], Theorem I):

Theorem 4.2 (Swan-Vigneras). $\mathbf{Z}\left[Q\left(2^{n}\right)\right]$ has the cancellation property for free modules only for the two values $n=3,4$; for all $n \geq 5$ there exist stably free modules over $\mathbf{Z}\left[Q\left(2^{n}\right)\right]$ which are not free.

For $m \geq 2, Q(4 m)$ has free period 4, as is clear from the complete resolution $0 \rightarrow \mathbf{Z} \stackrel{\epsilon^{*}}{\rightarrow} \mathbf{Z}[Q] \stackrel{\partial_{3}}{\rightarrow} \mathbf{Z}[Q]^{2} \stackrel{\partial_{2}}{\rightarrow} \mathbf{Z}[Q]^{2} \stackrel{\partial_{1}}{\rightarrow} \mathbf{Z}[Q] \stackrel{\epsilon}{\rightarrow} \mathbf{Z} \rightarrow 0$ where $\epsilon^{*}$ is the dual of the augmentation map, $\partial_{1}=(x-1, y-1)$,

$$
\partial_{2}=\left(\begin{array}{cc}
1+x+\ldots+x^{m-1} & y x+1 \\
-(y+1) & x-1
\end{array}\right) \text { and } \partial_{3}=\left(\begin{array}{c}
x-1 \\
1-y x
\end{array}\right) .
$$

In consequence we get:

Proposition 4.3. For all $m \geq 2, \mathbf{I}^{*}(Q(4 m))$ belongs to the minimal level of $\Omega_{3}(\mathbf{Z})$.

Proof. It is straightforward to see that $\operatorname{Im}\left(\partial_{3}\right) \cong \mathbf{I}^{*}(Q)$ so that, since $\operatorname{Ker}\left(\partial_{2}\right)=$ $\operatorname{Im}\left(\partial_{3}\right)$, the above resolution truncates to give an algebraic 2-complex of the form

$$
0 \rightarrow \mathbf{I}^{*}(Q) \rightarrow \mathbf{Z}[Q]^{2} \stackrel{\partial_{2}}{\rightarrow} \mathbf{Z}[Q]^{2} \stackrel{\partial_{1}}{\rightarrow} \mathbf{Z}[Q] \stackrel{\epsilon}{\rightarrow} \mathbf{Z} \rightarrow 0
$$

Minimality follows since $\operatorname{rk}_{\mathbf{Z}}\left(\mathbf{I}^{*}(Q)\right)<|Q|$. 
From (2.4), (4.1), (4.2) and (4.3) it follows that:

Corollary 4.4. Let $Q=Q\left(2^{n}\right)$ denote the generalised quaternion group of order $2^{n}$; then the following statements are equivalent:

(i) $Q$ is 1-straight;

(ii) $Q$ is 3-straight;

(iii) $n=3,4$.

The above corrects an over-optimistic opinion in [5] (p. 245, lines 7-9).

When $n \geq 5$, the fork $\operatorname{Alg}_{Q\left(2^{n}\right)}$ has at least two prongs, by Corollary 4.4(ii). Thus over $Q\left(2^{n}\right)$, the minimal level of $\Omega_{3}(\mathbf{Z})$ contains at least two distinct isomorphism classes, one of them being $\mathbf{I}^{*}\left(Q\left(2^{n}\right)\right)$. The algebraic 2-complex obtained by truncating the complete resolution is simply the Cayley complex of the presentation $\mathcal{Q}(4 m)$. In particular, it is geometrically realisable. Let $J \in \mathbf{A l g}_{\mathcal{Q}\left(2^{n}\right)}$ be an exotic root module, and let $\mathcal{X}_{*}$ be an algebraic 2 -complex such that $\pi_{2}\left(\mathcal{X}_{*}\right) \cong J$. Clearly $\mathcal{X}_{*} \nsucceq C_{*}\left(\mathcal{Q}\left(2^{n}\right)\right)$, since $J \not \mathbf{I}^{*}\left(Q\left(2^{n}\right)\right) \cong \pi_{2}\left(\mathcal{Q}\left(2^{n}\right)\right)$. Thus we obtain:

Theorem A. For each $n \geq 5$ there exists a minimal algebraic 2-complex $\mathcal{X}_{*}(n)$ over $Q\left(2^{n}\right)$ such that $\mathcal{X}_{*}(n)$ is not chain homotopy equivalent to $C_{*}\left(\mathcal{Q}\left(2^{n}\right)\right)$, the Cayley complex of the standard presentation.

It is well known [3] and easy to prove that if $\mathcal{G}=\left\langle x_{1}, \ldots, x_{g} \mid W_{1}, \ldots, W_{r}\right\rangle$ is a presentation of the finite group $G$, then $g \leq r$. The number $e(\mathcal{G})=r-g \geq 0$ is called the excess of $\mathcal{G}$; the presentation $\mathcal{G}$ is balanced when $e(\mathcal{G})=0$, and is minimal when $e(\mathcal{G}) \leq e(\mathcal{P})$ for any other finite presentation $\mathcal{P}$ of $G$.

In the example over $Q\left(2^{n}\right)$ considered above, it is not clear whether the minimal algebraic 2-complex $\chi_{*}(n)$ is realisable as $C_{*}(\mathcal{G})$ for some (necessarily balanced) presentation $\mathcal{G}$ of $Q\left(2^{n}\right)$. It follows from the main result of [6], however, that if $\chi_{*}(n)$ is not realisable, then the $\mathrm{D}(2)$-property fails for $Q\left(2^{n}\right)$; that is:

Theorem B. For each $n \geq 5$, either $Q\left(2^{n}\right)$ possesses a balanced presentation which is homotopically distinct from the standard presentation or else the $\mathrm{D}(2)$-property fails for $Q\left(2^{n}\right)$.

We note that if $\mathcal{P}, \mathcal{Q}$ are nonminimal finite presentations of the finite group $G$ with $e(\mathcal{P})=e(\mathcal{Q})$, then by Browning's Theorem $X_{\mathcal{P}} \simeq X_{\mathcal{Q}}$.

\section{REFERENCES}

1. W. Browning, Homotopy types of certain finite C.W. complexes with finite fundamental group. Ph.D. Thesis, Cornell University, 1978.

2. M. N. Dyer and A. J. Sieradski, Trees of homotopy types of two-dimensional $C W$-complexes. Comment. Math. Helv. 48 (1973), 31-44. MR 51:14074

3. D.B.A. Epstein, Finite presentations of groups and 3-manifolds. Quart. J. Math. Oxford Ser. (2) 12 (1961), 205-212. MR 26:1867

4. M. Gutierrez and M. P. Latiolais, Partial homotopy type of finite two-complexes. Math. Zeit. 207 (1991) 359-378. MR 92h:55007

5. F.E.A. Johnson, Stable modules and the structure of Poincaré 3-complexes. Geometry and Topology, Aarhus (Proceedings of the $6^{\text {th }}$ Aarhus Topology Conference). Contemp. Math. 258 (2000), 227-248. MR 2001e:57001

6. F.E.A. Johnson, Stable modules and Wall's D(2) problem. Comment. Math. Helv. 78 (2003), 18-44.

7. R. G. Swan, Periodic resolutions for finite groups. Ann. of Math. 72 (1960) 267-291. MR 23:A2205 
8. R. G. Swan, Projective modules over binary polyhedral groups. J. reine angew. Math. 342 (1983) 66-172. MR 84j:16003

9. M. F. Vigneras, Simplification pour les ordres des corps de quaternions totalement définis. J. reine angew. Math. 286/287 (1976) 257-277. MR 55:2851

10. C.T.C. Wall, Finiteness conditions for CW-complexes. Ann. of Math. 81 (1965) 56-69. MR 30:1515

Department of Mathematics, University College London, Gower Street, London WC1E 6BT, United Kingdom

E-mail address: feaj@math.ucl.ac.uk 